\title{
CryoEM Studies of Adenovirus-Integrin and Adenovirus-Defensin Complexes Indicate Conformational Changes during Cell Entry
}

\author{
P.L. Stewart,* S. Lindert,* M. Silvestry, ${ }^{*}$ T.-M. Mullen, ${ }^{* *}$ J.G. Smith, ${ }^{* *}$ and G.R. Nemerow** \\ *Department of Molecular Physiology and Biophysics, Vanderbilt University Medical Center, 2215 \\ Garland Avenue, Nashville, TN 37232 \\ **Department of Immunology and Microbial Science, The Scripps Research Institute, La Jolla, CA \\ 92037
}

A growing number of viruses, including human adenovirus (HAdV), have been identified as using integrin as a receptor for cell entry. The HAdV penton base contains five flexible RGD-containing loops that interact with $\alpha \mathrm{v}$ integrins triggering receptor mediated endocytosis. We performed a cryoEM analysis of the complex of HAdV 12 with a soluble form of $\alpha v \beta 5$ integrin [1]. A data set with 2,499 particle images of the HAdV12/ $\alpha \mathrm{v} \beta 5$ complex was acquired on an FEI Polara $(300 \mathrm{kV}$; FEG) microscope. The final cryoEM structure has subnanometer $(8 \AA)$ resolution for the icosahedral capsid including the penton base and more moderate resolution $(27 \AA)$ for the integrin density above each penton base (Fig. 1A). Although each penton base has five RGD-containing loops, modeling with integrin crystal structures indicates that a maximum of four integrins can fit over any penton base. We conclude that the close spacing $(\sim 60 \AA)$ of the RGD protrusions precludes integrin binding to all of the RGD sites, in agreement with previous Biacore results [2]. The flexible penton-base RGD loops and incoherent averaging of less than five bound integrin molecules per penton base likely explain the moderate resolution observed for the integrin density.

There is controversy regarding the integrin conformation after interaction with an RGD-containing ligand. One model, the "extension" or "switchblade" model, proposes a straightening of the integrin extracellular domains after interaction with a ligand. Another model, the "deadbolt" model, suggests only small conformational changes in the integrin $\beta$-chain I domain upon RGD binding leaving the overall integrin conformation bent, as observed in integrin structures without ligands. Comparison of the cryoEM density with both bent and extended integrin models indicates much better agreement with an extended conformation in which the integrin $\alpha$ and $\beta$ chains extend away from the penton base (Fig. 1B,C).

The cryoEM structure of the HAdV12/ $\alpha \mathrm{v} \beta 5$ complex suggested that integrin binding to HAdV might predispose the penton base to release from the capsid, even in the absence of the host cell membrane and the low $\mathrm{pH}$ environment of the endosome. When the HAdV12/ $\alpha \mathrm{v} \beta 5$ structure is compared to the previous Ad5.F35 structure [3] and displayed such that the density is similar for the hexon capsid protein, then the penton base density appears significantly reduced in the HAdV12/ $\alpha v \beta 5$ complex (Fig. 2). We have proposed that when four integrins bind per penton base one RGD-loop might extend in the direction that could induce a conformational change in the penton base, effectively inducing an untwisting of the pentamer [1]. In a cryoEM study of the interaction of HAdV with a neutralizing defensin HD5 we deduced that the defensin peptide might effectively lock the penton base and fiber complex together and prevent conformational changes that are necessary for cell entry [4]. Together the Ad/integrin and Ad/defensin cryoEM studies indicate that fiber release is a critical step in cell entry and that fiber release is likely concurrent 
with a major conformational change in penton base. These conformational changes may be stimulated by interaction of penton base with multiple integrin heterodimers.

\section{References}

[1] S. Lindert et al., J. Virol. 83 (2009) 11491.

[2] C.Y. Chiu et al., J. Virol. 73 (1999) 6759.

[3] S.D. Saban et al., J. Virol. 80 (2006) 12049.

[4] J.G. Smith, M. Silvestry, S. Lindert, W. Lu, G.R. Nemerow, and P.L. Stewart, Submitted.

[5] This research was supported by NIH grants T32 GM008320 (MS), EY11431 (GRN), HL054352 (GRN), and AI042929 (PLS).
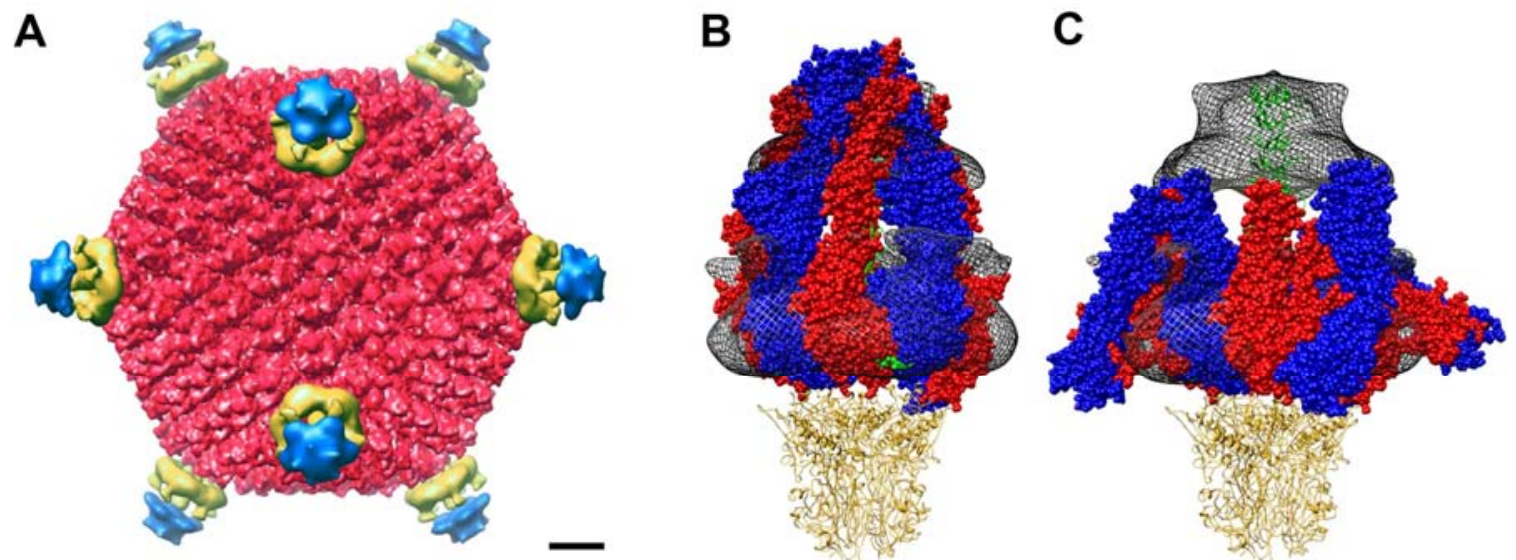

FIG.1. CryoEM structure of the HAdV12/ $\alpha v \beta 5$ integrin complex. (A) The structure is viewed along a twofold icosahedral axis with three radial shells: icosahedral capsid (300-463 , red), integrin ring

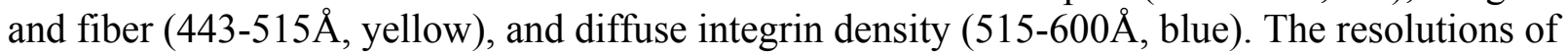
these shells are $8 \AA, 27 \AA$, and $85 \AA$, respectively (FSC 0.5 thresholds). Scale bar, $100 \AA$. (B) The cryoEM integrin density at one vertex (mesh) is shown with four integrin heterodimers in an extended conformation. (C) Similar to panel B, but with integrin heterodimers in a bent conformation. These are composite models for the integrin extracellular domains built from PDB $1 \mathrm{~L} 5 \mathrm{G}$ and $18 \mathrm{UC}$ with the $\alpha \mathrm{v}$ chains in blue, the $\beta$ chains in red, and the RGD peptide in green spheres. The penton base is shown in gold and the fiber in green ribbon.
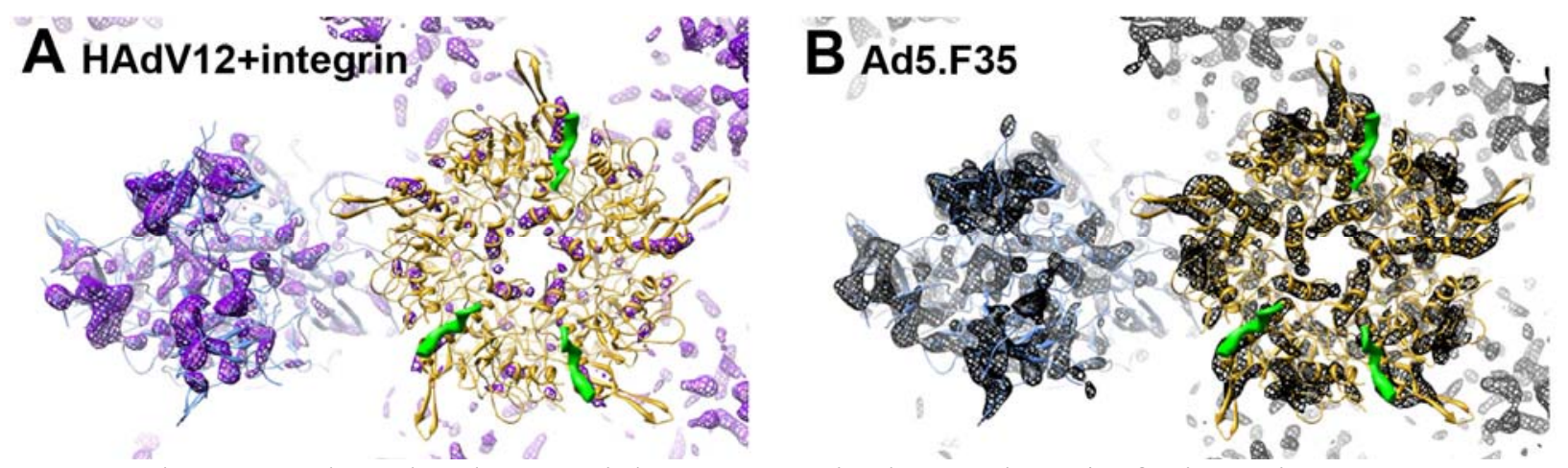

FIG. 2. The penton base has low partial occupancy in the HAdV12/ $\alpha \mathrm{v} \beta 5$ integrin cryoEM structure. (A) Enlarged view of a portion of the HAdV12/ $\alpha \mathrm{v} \beta 5$ structure (mesh) with docked crystal structures of hexon (blue) (PDB 1P30) and penton base (gold) with the N-terminal fiber peptide (green) (PDB 1X9T). (B) Similar to panel A, but with the Ad5.F35 cryoEM structure [3] for comparison. 